\title{
EXTENSION OF SOME CHENEY-SHARMA TYPE OPERATORS TO A TRIANGLE WITH ONE CURVED SIDE
}

\author{
TEODORA CĂTINAŞ
}

Received 28 September, 2018

\begin{abstract}
We extend the definition of some Cheney-Sharma type operators to a triangle with one curved side. We construct their product and Boolean sum, we study their interpolation properties, the orders of accuracy and we give different expressions of the corresponding remainders. We also give some illustrative examples.

2010 Mathematics Subject Classification: 41A35; 41A36; 41A25; 41A80

Keywords: Cheney-Sharma operator, product and Boolean sum operators, modulus of continuity, degree of exactness, the Peano's theorem, error evaluation
\end{abstract}

\section{INTRODUCTION}

In order to match all the boundary information on a curved domain (as Dirichlet, Neumann or Robin boundary conditions for differential equation problems), there were considered interpolation operators on domains with curved sides (see, e.g., [3], [4], [5], [6], [8], [9], [10]).

The aim of this paper is to construct some Cheney-Sharma type operators defined on a triangle with one curved side and to study the interpolation properties, the orders of accuracy and the remainders of the corresponding approximation formulas.

Using the interpolation properties of such operators, blending function interpolants can be constructed, that exactly match the function on some sides of the given region. Important applications of these blending functions are in computer aided geometric design, in finite element method for differential equations problems and for construction of surfaces which satisfy some given conditions.

\section{UNIVARIATE OPERATORS}

Let $m \in \mathbb{N}$ and $\beta$ be a nonnegative parameter. The Cheney-Sharma operator of second kind $Q_{m}: C[0,1] \rightarrow C[0,1]$, introduced in [7], is given by

$$
\left(Q_{m} f\right)(x)=\sum_{i=0}^{m} q_{m, i}(x) f\left(\frac{k}{m}\right)
$$


with

$$
q_{m, i}(x)=\left(\begin{array}{c}
m \\
i
\end{array}\right) \frac{x(x+i \beta)^{i-1}(1-x)[1-x+(m-i) \beta]^{m-i-1}}{(1+m \beta)^{m-1}} .
$$

We recall some results regarding these Cheney-Sharma type operators, studied, for example, in [1], [14].

Remark 1. 1) Notice that for $\beta=0$, the operator $Q_{m}$ becomes the Bernstein operator.

2) In [14], it has been proved that the Cheney-Sharma operator $Q_{m}$ interpolates a given function at the endpoints of the interval.

3) In [7] and [14], it has been proved that the Cheney-Sharma operator $Q_{m}$ reproduces the constant and the linear functions, so its degree of exactness is 1 (denoted $\left.\operatorname{dex}\left(Q_{m}\right)=1\right)$.

4) In [7] it is given the following result

$$
\begin{aligned}
\left(Q_{m} e_{2}\right)(x)= & x(1+m \beta)^{1-m}[S(2, m-2, x+2 \beta, 1-x) \\
& -(m-2) \beta S(2, m-3, x+2 \beta, 1-x+\beta)],
\end{aligned}
$$

where $e_{i}(x)=x^{i}, i \in \mathbb{N}$, and

$$
S(j, m, x, y)=\sum_{k=0}^{m}\left(\begin{array}{c}
m \\
k
\end{array}\right)(x+k \beta)^{k+j-1}[y+(m-k) \beta]^{m-k},
$$

$j=\overline{0, m}, m \in \mathbb{N}, x, y \in[0,1], \beta>0$.

We consider the standard triangle $\tilde{T}_{h}$ (see Figure 1), with vertices $V_{1}=(0, h), V_{2}=$ $(h, 0)$ and $V_{3}=(0,0)$, with two straight sides $\Gamma_{1}, \Gamma_{2}$, along the coordinate axes, and with the third side $\Gamma_{3}$ (opposite to the vertex $V_{3}$ ) defined by the one-to-one functions $f$ and $g$, where $g$ is the inverse of the function $f$, i.e., $y=f(x)$ and $x=g(y)$, with $f(0)=g(0)=h$, for $h>0$. Also, we have $f(x) \leq h$ and $g(y) \leq h$, for $x, y \in[0, h]$.

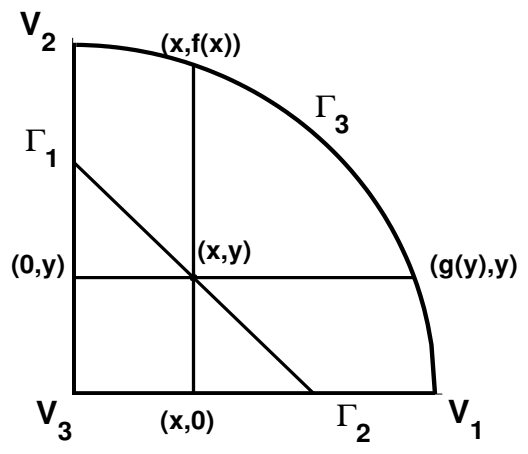

FIGURE 1. Triangle $\tilde{T}_{h}$. 
For $m, n \in \mathbb{N}, \beta, b \in \mathbb{R}_{+}$, we consider the following extensions of the CheneySharma operator given in (2.1):

$$
\begin{aligned}
& \left(Q_{m}^{x} F\right)(x, y)=\sum_{i=0}^{m} q_{m, i}(x, y) F\left(i \frac{g(y)}{m}, y\right), \\
& \left(Q_{n}^{y} F\right)(x, y)=\sum_{j=0}^{n} q_{n, j}(x, y) F\left(x, j \frac{f(x)}{n}\right),
\end{aligned}
$$

with

$$
\begin{aligned}
& q_{m, i}(x, y)=\left(\begin{array}{c}
m \\
i
\end{array}\right) \frac{\frac{x}{g(y)}\left(\frac{x}{g(y)}+i \beta\right)^{i-1}\left(1-\frac{x}{g(y)}\right)\left[1-\frac{x}{g(y)}+(m-i) \beta\right]^{m-i-1}}{(1+m \beta)^{m-1}}, \\
& q_{n, j}(x, y)=\left(\begin{array}{c}
n \\
j
\end{array}\right) \frac{\frac{y}{f(x)}\left(\frac{y}{f(x)}+j b\right)^{j-1}\left(1-\frac{y}{f(x)}\right)\left[1-\frac{y}{f(x)}+(n-j) b\right]^{n-j-1}}{(1+n b)^{n-1}},
\end{aligned}
$$

where

$$
\Delta_{m}^{x}=\left\{i \frac{g(y)}{m} \mid i=\overline{0, m}\right\} \text { and } \Delta_{n}^{y}=\left\{j \frac{f(x)}{n} \mid j=\overline{0, n}\right\}
$$

are uniform partitions of the intervals $[0, g(y)]$ and $[0, f(x)]$.

Remark 2. As the Cheney-Sharma operator of second kind interpolates a given function at the endpoints of the interval, we may use the operators $Q_{m}^{x}$ and $Q_{n}^{y}$ as interpolation operators.

Theorem 1. If $F$ is a real-valued function defined on $\widetilde{T}_{h}$ then

(i) $Q_{m}^{x} F=F$ on $\Gamma_{1} \cup \Gamma_{3}$,

(ii) $Q_{n}^{y} F=F$ on $\Gamma_{2} \cup \Gamma_{3}$.

Proof. (i) We may write

$$
\begin{aligned}
\left(Q_{m}^{x} F\right)(x, y)= & \frac{1}{(1+m \beta)^{m-1}}\left\{\left(1-\frac{x}{g(y)}\right)\left[1-\frac{x}{g(y)}+m \beta\right]^{m-1} F(0, y)\right. \\
& +\frac{x}{g(y)}\left(1-\frac{x}{g(y)}\right) \sum_{i=1}^{m-1}\left(\begin{array}{c}
m \\
i
\end{array}\right)\left(\frac{x}{g(y)}+i \beta\right)^{i-1} \\
& \cdot\left[1-\frac{x}{g(y)}+(m-i) \beta\right]^{m-i-1} F\left(i \frac{g(y)}{m}, y\right) \\
& \left.+\frac{x}{g(y)}\left(\frac{x}{g(y)}+m \beta\right)^{m-1} F(g(y), y)\right\} .
\end{aligned}
$$

Considering (2.5), we may easily prove that

$$
\begin{aligned}
& \left(Q_{m}^{x} F\right)(0, y)=F(0, y), \\
& \left(Q_{m}^{x} F\right)(g(y), y)=F(g(y), y) .
\end{aligned}
$$

(ii) Similarly, writing

$$
\left(Q_{n}^{y} F\right)(x, y)=\frac{1}{(1+n b)^{n-1}}\left\{\left(1-\frac{y}{f(x)}\right)\left[1-\frac{y}{f(x)}+n b\right]^{n-1} F(x, 0)\right.
$$




$$
\begin{aligned}
& +\frac{y}{f(x)}\left(1-\frac{y}{f(x)}\right) \sum_{j=1}^{n-1}\left(\begin{array}{l}
n \\
j
\end{array}\right)\left(\frac{y}{f(x)}+j b\right)^{j-1} \\
& \cdot\left[1-\frac{y}{f(x)}+(n-j) b\right]^{n-j-1} F\left(x, j \frac{f(x)}{n}\right) \\
& \left.+\frac{y}{f(x)}\left(\frac{y}{f(x)}+n b\right)^{n-1} F(x, f(x))\right\},
\end{aligned}
$$

we get that

$$
\begin{aligned}
& \left(Q_{n}^{y} F\right)(x, 0)=F(x, 0), \\
& \left(Q_{n}^{y} F\right)(x, f(x))=F(x, f(x)) .
\end{aligned}
$$

Theorem 2. The operators $Q_{m}^{x}$ and $Q_{n}^{y}$ have the following orders of accuracy:

(i) $\left(Q_{m}^{x} e_{i j}\right)(x, y)=x^{i} y^{j}, i=0,1 ; j \in \mathbb{N}$;

(ii) $\left(Q_{n}^{y} e_{i j}\right)(x, y)=x^{i} y^{j}, i \in \mathbb{N} ; j=0,1$, where $e_{i j}(x, y)=x^{i} y^{j}, i, j \in \mathbb{N}$.

Proof. (i) We have

$$
\left(Q_{m}^{x} e_{i j}\right)(x, y)=y^{j} \sum_{i=0}^{m} q_{m, i}(x, y)\left[i \frac{g(y)}{m}\right]^{i},
$$

and since the degree of exactness of the univariate Cheney-Sharma operator is equal to 1 (see Remark 1), the result follows.

Property (ii) is proved in the same way.

We consider the approximation formula

$$
F=Q_{m}^{x} F+R_{m}^{x} F
$$

where $R_{m}^{x} F$ denotes the approximation error.

Theorem 3. If $F(\cdot, y) \in C[0, g(y)]$ then we have

$$
\left|\left(R_{m}^{x} F\right)(x, y)\right| \leq\left(1+\frac{1}{\delta} \sqrt{A_{m}-x^{2}}\right) \omega(F(\cdot, y) ; \delta), \quad \forall \delta>0,
$$

where $\omega(F(\cdot, y) ; \delta)$ is the modulus of continuity and $A_{m}=x(1+m \beta)^{1-m}[S(2, m-$ $2, x+2 \beta, 1-x)-(m-2) \beta S(2, m-3, x+2 \beta, 1-x+\beta)]$, with $S$ given in (2.3).

Proof. By Theorem 2 we have that $\operatorname{dex}\left(Q_{m}^{x}\right)=1$, thus we may apply the following property of linear operators (see, for example, [2], [13]):

$$
\left|\left(Q_{m}^{x} F\right)(x, y)-F(x, y)\right| \leq\left[1+\delta^{-1} \sqrt{\left(Q_{m}^{x} e_{20}\right)(x, y)-x^{2}}\right] \omega(F(\cdot, y) ; \delta), \quad \forall \delta>0,
$$

and taking into account (2.2), we get (2.6).

Theorem 4. If $F(\cdot, y) \in C^{2}[0, g(y)]$ then

$$
\begin{aligned}
\left(R_{m}^{x} F\right)(x, y)= & \frac{1}{2} F^{(2,0)}(\xi, y)\left\{x^{2}-x(1+m \beta)^{1-m}[S(2, m-2, x+2 \beta, 1-x)\right. \\
& -(m-2) \beta S(2, m-3, x+2 \beta, 1-x+\beta)]\},
\end{aligned}
$$


for $\xi \in[0, g(y)]$ and $\beta>0$.

Proof. Taking into account that $\operatorname{dex}\left(Q_{m}^{x}\right)=1$, by Theorem 2, and applying the Peano's theorem (see, e.g., [12]), it follows

$$
\left(R_{m}^{x} F\right)(x, y)=\int_{0}^{g(y)} K_{20}(x, y ; s) F^{(2,0)}(s, y) d s,
$$

where

$$
K_{20}(x, y ; s)=(x-s)_{+}-\sum_{i=0}^{m} q_{m, i}(x, y)\left(i \frac{g(y)}{m}-s\right)_{+} .
$$

For a given $v \in\{1, \ldots, m\}$ one denotes by $K_{20}^{v}(x, y ; \cdot)$ the restriction of the kernel $K_{20}(x, y ; \cdot)$ to the interval $\left[(\nu-1) \frac{g(y)}{m}, v \frac{g(y)}{m}\right]$, i.e.,

$$
K_{20}^{v}(x, y ; v)=(x-s)_{+}-\sum_{i=v}^{m} q_{m, i}(x, y)\left(i \frac{g(y)}{m}-s\right),
$$

whence,

$$
K_{20}^{v}(x, y ; s)= \begin{cases}x-s-\sum_{i=v}^{m} q_{m, i}(x, y)\left(i \frac{g(y)}{m}-s\right), & s<x \\ -\sum_{i=v}^{m} q_{m, i}(x, y)\left(i \frac{g(y)}{m}-s\right), & s \geq x .\end{cases}
$$

It follows that $K_{20}^{v}(x, y ; s) \leq 0$, for $s \geq x$.

For $s<x$ we have

$$
\begin{aligned}
K_{20}^{v}(x, y ; s)= & x-s-\sum_{i=0}^{m} q_{m, i}(x, y)\left[i \frac{g(y)}{m}-s\right] \\
& +\sum_{i=0}^{v-1} q_{m, i}(x, y)\left[i \frac{g(y)}{m}-s\right] .
\end{aligned}
$$

Applying Theorem 2, we get

$$
\sum_{i=0}^{m} q_{m, i}(x, y)\left[i \frac{g(y)}{m}-s\right]=\left(Q_{m}^{x} e_{10}\right)(x, y)-s\left(Q_{m}^{x} e_{00}\right)(x, y)=x-s,
$$

whence it follows that

$$
K_{20}^{v}(x, y ; s)=\sum_{i=0}^{v-1} q_{m, i}(x, y)\left[i \frac{g(y)}{m}-s\right] \leq 0 .
$$

So, $K_{20}^{v}(x, y ; \cdot) \leq 0$, for any $v \in\{1, \ldots, m\}$, i.e., $K_{20}(x, y ; s) \leq 0$, for $s \in[0, g(y)]$.

By the Mean Value Theorem, one obtains

$$
\left(R_{m}^{x} F\right)(x, y)=F^{(2,0)}(\xi, y) \int_{0}^{g(y)} K_{20}(x, y ; s) d s, \text { for } 0 \leq \xi \leq g(y),
$$


with

$$
\int_{0}^{g(y)} K_{20}(x, y ; s) d s=\frac{1}{2}\left[x^{2}-\left(Q_{m}^{x} e_{20}\right)(x, y)\right],
$$

and using (2.2) we get (2.7).

Remark 3. Analogous results with the ones in Theorems 3 and 4 could be obtained for the remainder $R_{n}^{y} F$ of the formula $F=Q_{n}^{y} F+R_{n}^{y} F$.

\section{PROduct OPERATORS}

Let $P_{m n}^{1}=Q_{m}^{x} Q_{n}^{y}$, respectively, $P_{n m}^{2}=Q_{n}^{y} Q_{m}^{x}$ be the products of the operators $Q_{m}^{x}$ and $Q_{n}^{y}$.

We have

$$
\left(P_{m n}^{1} F\right)(x, y)=\sum_{i=0}^{m} \sum_{j=0}^{n} q_{m, i}(x, y) q_{n, j}\left(i \frac{g(y)}{m}, y\right) F\left(i \frac{g(y)}{m}, j \frac{f\left(i \frac{g(y)}{m}\right)}{n}\right),
$$

respectively,

$$
\left(P_{n m}^{2} F\right)(x, y)=\sum_{i=0}^{m} \sum_{j=0}^{n} q_{m, i}\left(x, j \frac{f(x)}{n}\right) q_{n, j}(x, y) F\left(i \frac{g\left(j \frac{f(x)}{n}\right)}{m}, j \frac{f(x)}{n}\right) .
$$

Theorem 5. If $F$ is a real-valued function defined on $\widetilde{T}_{h}$ then

(i) $\left(P_{m n}^{1} F\right)\left(V_{i}\right)=F\left(V_{i}\right), \quad i=1,2,3$;

$\left(P_{m n}^{1} F\right)\left(\Gamma_{3}\right)=F\left(\Gamma_{3}\right)$,

(ii) $\left(P_{n m}^{2} F\right)\left(V_{i}\right)=F\left(V_{i}\right), \quad i=1,2,3$;

$$
\left(P_{n m}^{2} F\right)\left(\Gamma_{3}\right)=F\left(\Gamma_{3}\right) \text {, }
$$

Proof. By a straightforward computation, we get the following properties

$$
\begin{aligned}
& \left(P_{m n}^{1} F\right)(x, 0)=\left(Q_{m}^{x} F\right)(x, 0), \\
& \left(P_{m n}^{1} F\right)(0, y)=\left(Q_{n}^{y} F\right)(0, y), \\
& \left(P_{m n}^{1} F\right)(x, f(x))=F(x, f(x)), \quad x, y \in[0, h]
\end{aligned}
$$

and

$$
\begin{aligned}
& \left(P_{n m}^{2} F\right)(x, 0)=\left(Q_{m}^{x} F\right)(x, 0), \\
& \left(P_{n m}^{2} F\right)(0, y)=\left(Q_{n}^{y} F\right)(0, y), \\
& \left(P_{n m}^{2} F\right)(g(y), y)=F(g(y), y), \quad x, y \in[0, h],
\end{aligned}
$$

and, taking into account Theorem 1, they imply (i) and (ii).

We consider the following approximation formula

$$
F=P_{m n}^{1} F+R_{m n}^{P^{1}} F,
$$

where $R_{m n}^{P^{1}}$ is the corresponding remainder operator. 
Theorem 6. If $F \in C\left(\widetilde{T}_{h}\right)$ then

$$
\left|\left(R_{m n}^{P^{1}} F\right)(x, y)\right| \leq\left(A_{m}+B_{n}-x^{2}-y^{2}+1\right) \omega\left(F ; \frac{1}{\sqrt{A_{m}-x^{2}}}, \frac{1}{\sqrt{B_{n}-y^{2}}}\right), \forall(x, y) \in \widetilde{T}_{h},
$$

where

$$
\begin{aligned}
A_{m}= & x(1+m \beta)^{1-m}[S(2, m-2, x+2 \beta, 1-x) \\
& -(m-2) \beta S(2, m-3, x+2 \beta, 1-x+\beta)] \\
B_{n}= & y(1+n b)^{1-n}[S(2, n-2, y+2 b, 1-y) \\
& -(n-2) b S(2, n-3, y+2 b, 1-y+\beta)]
\end{aligned}
$$

and $\omega\left(F ; \delta_{1}, \delta_{2}\right)$, with $\delta_{1}>0, \delta_{2}>0$, is the bivariate modulus of continuity.

Proof. Using a basic property of the modulus of continuity we have

$$
\begin{aligned}
\left|\left(R_{m n}^{P^{1}} F\right)(x, y)\right| \leq & {\left[\frac{1}{\delta_{1}} \sum_{i=0}^{m} \sum_{j=0}^{n} q_{m, i}(x, y) q_{n, j}\left(\frac{i}{m} g(y), y\right)\left|x-\frac{i}{m} g(y)\right|\right.} \\
& +\frac{1}{\delta_{2}} \sum_{i=0}^{m} \sum_{j=0}^{n} q_{m, i}(x, y) q_{n, j}\left(\frac{i}{m} g(y), y\right)\left|y-\frac{j}{n} f\left(\frac{i}{m} g(y)\right)\right| \\
& \left.+\sum_{i=0}^{m} \sum_{j=0}^{n} q_{m, i}(x, y) q_{n, j}\left(\frac{i}{m} g(y), y\right)\right] \omega\left(F ; \delta_{1}, \delta_{2}\right), \forall \delta_{1}, \delta_{2}>0 .
\end{aligned}
$$

Since

$$
\begin{aligned}
& \sum_{i=0}^{m} \sum_{j=0}^{n} p_{m, i}(x, y) q_{n, j}\left(\frac{i}{m} g(y), y\right)\left|x-\frac{i}{m} g(y)\right| \leq \sqrt{\left(Q_{m}^{x} e_{20}\right)(x, y)-x^{2}}, \\
& \sum_{i=0}^{m} \sum_{j=0}^{n} p_{m, i}(x, y) q_{n, j}\left(\frac{i}{m} g(y), y\right)\left|y-\frac{j}{n} f\left(\frac{i}{m} g(y)\right)\right| \leq \sqrt{\left(Q_{n}^{y} e_{02}\right)(x, y)-y^{2}} \\
& \sum_{i=0}^{m} \sum_{j=0}^{n} p_{m, i}(x, y) q_{n, j}\left(\frac{i}{m} g(y), y\right)=1,
\end{aligned}
$$

applying (2.2), we get

$$
\begin{aligned}
& \left|\left(R_{m n}^{P^{1}} F\right)(x, y)\right| \leq\left\{\frac{1}{\delta_{1}}\left[x(1+m \beta)^{1-m}\right]^{\frac{1}{2}}\right. \\
& \cdot\{[S(2, m-2, x+2 \beta, 1-x) \\
& \left.-(m-2) \beta S(2, m-3, x+2 \beta, 1-x+\beta)]-x^{2}\right\}^{\frac{1}{2}}+\frac{1}{\delta_{2}}\left[y(1+n b)^{1-n}\right]^{\frac{1}{2}} \\
& \cdot\{[S(2, n-2, y+2 b, 1-y) \\
& \left.\left.-(n-2) b S(2, n-3, y+2 b, 1-y+\beta)]-y^{2}\right\}^{\frac{1}{2}}+1\right\} \omega\left(F ; \delta_{1}, \delta_{2}\right) .
\end{aligned}
$$

Denoting

$$
A_{m}=x(1+m \beta)^{1-m}[S(2, m-2, x+2 \beta, 1-x)
$$




$$
\begin{aligned}
& -(m-2) \beta S(2, m-3, x+2 \beta, 1-x+\beta)] \\
B_{n}= & y(1+n b)^{1-n}[S(2, n-2, y+2 b, 1-y) \\
& -(n-2) b S(2, n-3, y+2 b, 1-y+\beta)]
\end{aligned}
$$

and, taking $\delta_{1}=\frac{1}{\sqrt{A_{m}-x^{2}}}$ and $\delta_{2}=\frac{1}{\sqrt{B_{n}-y^{2}}}$, we get (3.1).

\section{BOOLEAN SUM OPERATORS}

We consider the Boolean sums of the operators $Q_{m}^{x}$ and $Q_{n}^{y}$,

$$
\begin{aligned}
& S_{m n}^{1}:=Q_{m}^{x} \oplus Q_{n}^{y}=Q_{m}^{x}+Q_{n}^{y}-Q_{m}^{x} Q_{n}^{y}, \\
& S_{n m}^{2}:=Q_{n}^{y} \oplus Q_{m}^{x}=Q_{n}^{y}+Q_{m}^{x}-Q_{n}^{y} Q_{m}^{x} .
\end{aligned}
$$

Theorem 7. If $F$ is a real-valued function defined on $\widetilde{T}_{h}$, then

$$
\begin{aligned}
& \left.S_{m n}^{1} F\right|_{\partial \widetilde{T}_{h}}=\left.F\right|_{\partial \widetilde{T}_{h}}, \\
& \left.S_{m n}^{2} F\right|_{\partial \widetilde{T}_{h}}=\left.F\right|_{\partial \widetilde{T}_{h}} .
\end{aligned}
$$

Proof. We have

$$
\begin{aligned}
\left(Q_{m}^{x} Q_{n}^{y} F\right)(x, 0) & =\left(Q_{m}^{x} F\right)(x, 0), \\
\left(Q_{n}^{y} Q_{m}^{x} F\right)(0, y) & =\left(Q_{n}^{y} F\right)(0, y), \\
\left(Q_{m}^{x} F\right)(x, h-x) & =\left(Q_{n}^{y} F\right)(x, h-x) \\
& =\left(P_{m n}^{1} F\right)(x, h-x)=\left(P_{n m}^{2} F\right)(x, h-x)=F(x, h-x),
\end{aligned}
$$

and, taking into account Theorem 1, the conclusion follows.

We consider the following approximation formula

$$
F=S_{m n}^{1} F+R_{m n}^{S^{1}} F
$$

where $R_{m n}^{s^{1}}$ is the corresponding remainder operator.

Theorem 8. If $F \in C\left(\widetilde{T}_{h}\right)$ then

$$
\begin{aligned}
& \left|\left(R_{m n}^{s^{1}} F\right)(x, y)\right| \leq \\
& \leq\left(1+A_{m}-x^{2}\right) \omega\left(F(\cdot, y) ; \frac{1}{\sqrt{A_{m}-x^{2}}}\right)+\left(1+B_{n}-y^{2}\right) \omega\left(F(x, \cdot) ; \frac{1}{\sqrt{B_{n}-y^{2}}}\right) \\
& +\left(A_{m}+B_{n}-x^{2}-y^{2}+1\right) \omega\left(F ; \frac{1}{\sqrt{A_{m}-x^{2}}}, \frac{1}{\sqrt{B_{n}-y^{2}}}\right),
\end{aligned}
$$

with $A_{m}$ and $B_{n}$ given in (3.2). 
Proof. The identity

$$
F-S_{m n}^{1} F=\left(F-Q_{m}^{x} F\right)+\left(F-Q_{n}^{y} F\right)-\left(F-P_{m n}^{1} F\right)
$$

implies that

$$
\left|\left(R_{m n}^{S^{1}} F\right)(x, y)\right| \leq\left|\left(R_{m}^{x} F\right)(x, y)\right|+\left|\left(R_{n}^{y} F\right)(x, y)\right|+\left|\left(R_{m n}^{P^{1}} F\right)(x, y)\right|,
$$

and, applying Theorems 3 and 6, we get (4.1).

\section{NUMERICAL EXAMPLES}

We consider the function:

Gentle: $\quad F(x, y)=\frac{1}{3} \exp \left[-\frac{81}{16}\left((x-0.5)^{2}+(y-0.5)^{2}\right)\right]$,

generally used in the literature, (see, e.g., [11]). In Figure 2 we plot the graphs of $F$, $Q_{m}^{x} F, Q_{n}^{y} F, P_{m n}^{1} F, S_{m n}^{1} F$, on $\tilde{T}_{h}$, considering $h=1, m=5, n=6, \beta=1$ and we can see the good approximation properties. 

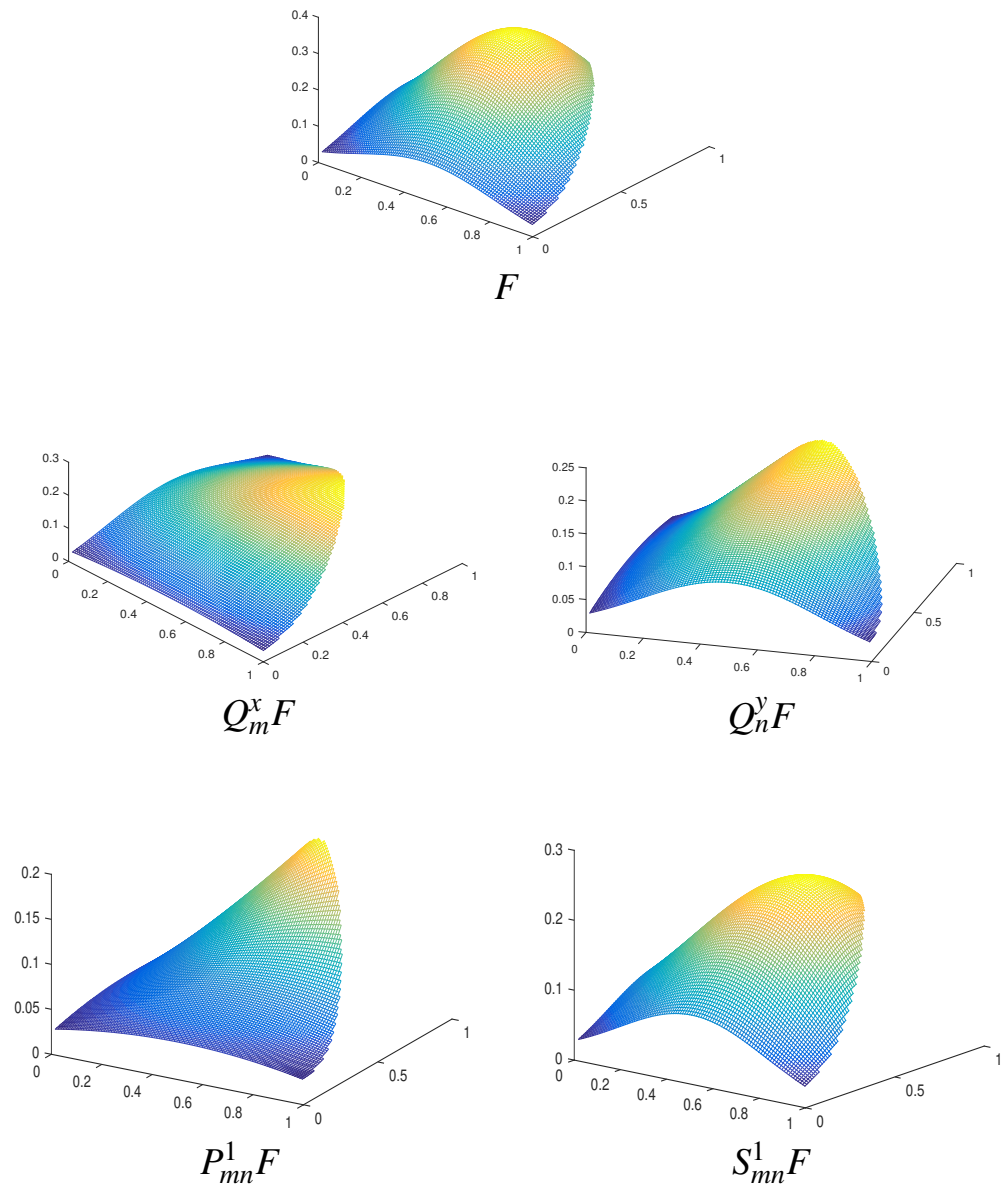

FIGURE 2. The Cheney-Sharma approximants for $\tilde{T}_{h}$.

\section{REFERENCES}

[1] O. Agratini, "Linear operators generated by a probability density function." Advances in Constructive Approximation: Vanderbilt (Eds., M. Neamtu and E. B. Saff); Proc. of International Conference Advances in Constructive Approximation, 14-17 May, 2003, Nashville, Tennessee, Nashboro Press, TN, 2004, pp. 1-12, 2003.

[2] O. Agratini, Approximation by linear operators. Cluj-Napoca: Cluj University Press, 2010.

[3] R. E. Barnhill and J. A. Gregory, "Compatible smooth interpolation in triangles." J. Approx. Theory, vol. 15 , pp. 214-225, 1975.

[4] P. Blaga, T. Cătinaş, and G. Coman, "Bernstein-type operators on triangle with all curved sides." Appl. Math. Comput., vol. 218, pp. 3072-3082, 2011. 
[5] P. Blaga, T. Cătinaş, and G. Coman, "Bernstein-type operators on triangle with one curved side." Mediterr. J. Math., vol. 9, no. 4, pp. 843-855, 2012.

[6] T. Catinaş, "Extension of some particular interpolation operators to a triangle with one curved side." Appl. Math. Comput., vol. 315, pp. 286-297, 2017.

[7] E. Cheney and A. Sharma, "On a generalization of Bernstein polynomials." Riv. Mat. Univ. Parma, vol. 5, pp. 77-84, 1964.

[8] G. Coman and T. Cătinaş, "Interpolation operators on a triangle with one curved side." BIT Numer. Math., vol. 50, no. 2, pp. 243-267, 2010.

[9] J. A. Marshall and A. R. Mitchell, "An exact boundary tehnique for improved accuracy in the finite element method." J. Inst. Maths. Applics., vol. 12, pp. 355-362, 1973.

[10] A. R. Mitchell and R. McLeod, "Curved elements in the finite element method." Conference on Numer. Sol.Diff. Eq., Lectures Notes in Mathematics, vol. 363, pp. 89-104, 1974

[11] R. J. Renka and A. K. Cline, “A triangle-based $C^{1}$ interpolation method." Rocky Mountain J. Math., vol. 14, pp. 223-237, 1984.

[12] A. Sard, Linear Approximation. Providence, Rhode Island: American Mathematical Society, 1963.

[13] O. Shisha and B. Mond, " The degree of convergence of linear positive operators." Proc. Nat. Acad. Sci. , vol. 60, pp. 1196-1200, 1968.

[14] D. D. Stancu and C. Cişmaşiu, "On an approximating linear positive operator of Cheney-Sharma." Rev. Anal. Numer. Theor. Approx., vol. 26, pp. 221-227, 1997.

Author's address

Teodora Cătinaş

Babeş-Bolyai University, Faculty of Mathematics and Computer Science, 1 M. Kogălniceanu St., RO-400084 Cluj-Napoca, Romania

E-mail address: tcatinas@math.ubbcluj.ro 